\title{
COMBINATORIAL SYMMETRY OF LINE ARRANGEMENTS AND APPLICATIONS
}

\author{
MEIRAV AMRAM, MOSHE COHEN, HAO SUN, MINA TEICHER, FEI YE, AND ANNA ZARKH
}

\begin{abstract}
We introduce an algorithm that exploits a combinatorial symmetry of an arrangement in order to produce a geometric reflection between two disconnected components of its moduli space. We apply this method to disqualify three real examples found in previous work by the authors from being Zariski pairs. Robustness is shown by its application to complex cases, as well.
\end{abstract}

\section{INTRODUCTION}

A line arrangement $\mathcal{A}=\left\{L_{1}, \ldots, L_{n}\right\}$ in $\mathbb{C P}^{2}$ is a finite collection of projective lines. The set $\operatorname{Lat}(\mathcal{A})=\left\{\bigcap_{i \in S} L_{i} \mid S \subseteq\{1,2, \ldots, n\}\right\}$ partially ordered by reverse inclusion is called the intersection lattice of $\mathcal{A}$. Two line arrangements $\mathcal{A}$ and $\mathcal{B}$ are lattice isomorphic, denoted by $\mathcal{A} \sim \mathcal{B}$, if up to a permutation on the labels of the lines their lattices are the same. In this case we say that the arrangements have the same combinatorics.

A Zariski pair of line arrangements is a pair of lattice isomorphic arrangements $\mathcal{A} \sim \mathcal{B}$ that have different embeddings in $\mathbb{C P}^{2}$. This means that the pairs $\left(\mathbb{C P}^{2}, \mathcal{A}\right)$ and $\left(\mathbb{C P}^{2}, \mathcal{B}\right)$ are not homeomorphic. Rybnikov Ryb11 found the first such pair of arrangements in 1998 and showed furthermore that the complements have different fundamental groups. Artal Bartolo, Carmona Ruber, Cogolludo Agustín, and Marco Buzunáriz [ABCRCAMB05] give another example explicitly.

One necessary condition for a Zariski pair is a disconnected moduli space. We define the moduli space of an arrangement $\mathcal{A}$ to be

$$
\left.\mathcal{M}_{\mathcal{A}}=\left\{\mathcal{B} \in\left(\left(\mathbb{C P}^{2}\right)^{*}\right)^{n} \mid \mathcal{B} \sim \mathcal{A}\right)\right\} / P G L(3, \mathbb{C}) .
$$

By Randell's Isotopy Theorem [Ran89, the embedding types of arrangements in the same connected component are the same.

By studying moduli spaces, Nazir and Yoshinaga NY12 proved that there is no Zariski pair of arrangements of up to eight complex lines and listed a classification of arrangements of nine lines without proof (later proved to be complete by Ye in Ye13]). The classification implies that there is also no Zariski pair of arrangements of nine lines.

Following this methodology, a classification of the moduli spaces of arrangements of ten lines was completed by the authors in [ATY13] and ACTY13. By Theorem 5.3 and Corollary 5.5. in ACTY13, this gives a list of eighteen potential Zariski pairs, as determined via disconnected moduli spaces, for all complex line arrangements of ten lines satisfying a reasonable assumption [ACTY13, Assumption 1.2].

Just one of these arrangements has a moduli space of dimension one; the rest have dimension zero. Of these seventeen, only seven are realizable with real coefficients. Following the enumeration from [ACSTY13] and intentionally omitting the cases $\{2\}$ through $\{5\}$, we rename them as follows:

Date: November 8, 2018.

2010 Mathematics Subject Classification. 14N20, 52C35.

Key words and phrases. automorphism group, complement, embedding type, intersection lattice.

This work was partially supported by the Emmy Noether Research Institute for Mathematics of the Minerva Foundation of Germany, the Oswald Veblen Fund, the Institute for Advanced Study in Princeton, USA, and the Polytechnic Institute of New York University. 
\{1\}. Equation (1) from [ATY13, Theorem 4.4]

$\{6\} . \quad(93)$.iii.ACG. from [ACTY13, Lemma 8.4]

$\{7\}$. (93).iii.BDF. from [ACTY13, Lemma 8.4]

as we will consider them below. Specifically these three have moduli spaces that are two distinct points. Let $t^{ \pm}$be the two solutions of the quadratic defining equation giving the two disconnected components of the moduli space. Then we will refer to points of the moduli spaces throughout as + and - .

There are cases with moduli spaces that are two distinct points (or more general two disconnected components of any dimension) arising from complex conjugation $x \mapsto \bar{x}, y \mapsto \bar{y}$, and $z \mapsto \bar{z}$. These have already been removed from the list above due to the fact that embedding types of two complex conjugate arrangements are the same. Furthermore, Cohen and Suciu [CS97, Theorem 3.9] proved that the braid monodromies of complex conjugated curves are equivalent.

Motivated by the effect of complex conjugation acting on moduli spaces, our present results show the geometric symmetry $\varphi: x \mapsto y, y \mapsto x$, and $z \mapsto z$ can similarly be used to disqualify some potential Zariski pairs.

Results. This work relies on Algorithm 2.4 to produce this geometric reflection $\varphi$ by exploiting a combinatorial $\mathbb{Z}_{2}$ subgroup of the automorphism group of the arrangement.

We apply this technique to show that arrangements $\{1\},\{6\}$, and $\{7\}$ are not Zariski pairs, as stated in the Main Theorem 2.6. These three arrangements have automorphism groups which contain a $\mathbb{Z}_{2}$ subgroup; the omitted four do not.

Ramifications. We suspect that the authors of [ABCRCAMB05] were aware of this type of situation based on several comments, specifically in Examples 3.4, 3.5, 3.6, Remark 3.8, and again in Section 5. In fact they refer to our definition of the moduli space as the ordered moduli space.

In our work we pair previous geometric techniques used by Nazir and Yoshinaga to determine this (ordered) moduli space together with insight from the automorphism group. This gives us an understanding of the more general moduli space of Artal Bartolo et al. In particular, our algorithm gives insight into where such a situation might arise.

Organization. After some background definitions, Subsection 2.1 presents the Algorithm 2.4 that we apply to several cases, which are listed in Table 1 and considered in seperate sections below.

The new real cases $\{1\},\{6\}$, and $\{7\}$ are listed in the Main Theorem 2.6 and considered in more detail in Section 4 .

The complex cases are considered in Section 5 with Subsection 5.1 on two examples from the literature, the MacLane arrangement and the Nazir-Yoshinaga arrangement, and Subsection 5.2 on examples from [ACTY13. The geometric reflection given by complex conjugation can already be used to disqualify these as Zariski pairs, but our geometric reflection $\varphi$ works, as well.

Lastly Section 6 points out two examples from the literature, the Falk-Sturmfels arrangement and Rybnikov's example, that act as counterexamples to our Algorithm 2.4 as it stands now.

\section{Determining the GeOmetric Reflection via COMbinatorial Symmetry}

The combinatorics of line arrangements can be given by means of several equivalent objects: an intersection lattice, a line combinatorics (as seen in [ABCRCAMB05]), a combinatorial type (as seen in [ABCRCAMB06]), and a configuration table (as seen in [Grü09]), which omits double points as these can be recovered from points of higher multiplicities alone.

It is this last most concise expression, the configuration table, that we use to describe the combinatorics in the examples below. However, in order to define combinatorial symmetries, we keep the intersection lattice terminology: 
Definition 2.1. Let $\mathcal{A}=\left\{L_{1}, \ldots, L_{n}\right\}$ and $\mathcal{A}^{\prime}=\left\{L_{1}^{\prime}, \ldots, L_{n}^{\prime}\right\}$ be two line arrangements in $\mathbb{C P}^{2}$. A lattice isomorphism between $\operatorname{Lat}(\mathcal{A})$ and $\operatorname{Lat}\left(\mathcal{A}^{\prime}\right)$ is a permutation $\tau$ on the index set $\{1, \ldots, n\}$ for which $\tau(\operatorname{Lat}(\mathcal{A})):=\operatorname{Lat}\left(\left\{L_{\tau(1)}, \ldots, L_{\tau(n)}\right\}\right)$ is identical to $\operatorname{Lat}\left(\mathcal{A}^{\prime}\right)$.

Definition 2.2. Let Lat $(\mathcal{A})$ be the intersection lattice of a line arrangement. We denote by $\operatorname{Aut}(\mathcal{A})$ the automorphism group or group of symmetries of $\operatorname{Lat}(\mathcal{A})$ and define it as the group of all lattice isomorphisms of $\operatorname{Lat}(\mathcal{A})$.

Examples of the automorphism groups of arrangements appear throughout the paper. The unfamiliar reader will appreciate the proof of Proposition 4.2 which explains this idea in careful detail for arrangement $\{1\}$.

2.1. The main algorithm and results. We use the following algorithm to generate representatives of disconnected components of a moduli space $\mathcal{M}_{\mathcal{A}}$ that are mapped to each other by $\varphi$.

Notation 2.3. The $\varphi$ that appears throughout the paper refers to the reflection $\varphi: \mathbb{C P}^{2} \rightarrow \mathbb{C P}^{2}$ that sends $x \mapsto y, y \mapsto x$, and $z \mapsto z$.

Algorithm 2.4. Given an arrangement $\mathcal{A}$ with some $\mathbb{Z}_{2}$ subgroup of the automorphism group $\operatorname{Aut}(\mathcal{A})$, we apply the following steps:

(1) Choose a permutation $\sigma$ that generates a $\mathbb{Z}_{2}$ subgroup, and identify its action on the lines of the arrangement $\mathcal{A}$.

(2) Choose two lines $L_{i} \neq L_{j}$ such that $L_{\sigma(i)} \neq L_{i}, L_{j}$ and $L_{\sigma(j)} \neq L_{j}$. Set the lines $L_{i}, L_{j}$, $L_{\sigma(i)}$, and $L_{\sigma(j)}$ as the lines $x=0, x=z, y=0$, and $y=z$, respectively.

(3) Apply the Grid Lemma 3.10 of [ACTY13 and the same techniques of previous work to obtain a parametrized equation that defines representative arrangements from each of the connected components of the moduli space $\mathcal{M}_{\mathcal{A}}$.

(4) For an appropriate pair $\mathcal{A}=\left\{L_{1}, \ldots, L_{n}\right\}$ and $\mathcal{A}^{\prime}=\left\{L_{1}^{\prime}, \ldots, L_{n}^{\prime}\right\}$ of the obtained arrangements, check that the map $\varphi$ sends the line arrangement $\left\{L_{1}, \ldots, L_{n}\right\}$ to the line arrangement $\left\{L_{\sigma(1)}^{\prime}, \ldots, L_{\sigma(n)}^{\prime}\right\}$.

Remark 2.5. In the last part of the algorithm, the check must involve the parameters of the defining equation. Let $t^{ \pm}$be the two solutions of the quadratic giving the two disconnected components of the moduli space. Often the reflection $\varphi$ realizes the Galois conjugation $t^{ \pm} \mapsto t^{\mp}$ by means of an additional inverse operation $t^{ \pm} \mapsto\left(t^{\mp}\right)^{-1}$ on the parameter $t$ (up to some constant)!

A list of arrangements that we consider in this work can be found in Table 1. We apply our Algorithm 2.4 successfully to those in the first two sections of the table. See Main Theorem 2.6 below.

The first three arrangements are realizable with real coefficients, have two disconnected components, and have dimension zero. Let $t^{ \pm}$be the two solutions of the quadratic defining equation giving the two disconnected components of the moduli space. Then we will refer to points of the moduli spaces throughout as + and - .

The next five arrangements cannot be realized with real coefficients, have two disconnected components, and have dimension either zero (the first two) or one (the last three). In the two former cases, the variable $t^{ \pm}$stands as above, along with the notation + and - . In the three latter cases, the variable $s^{ \pm}$will give the two disconnected components of the moduli space, while the variable $t$ will act as the free variable giving dimension one. See Notation 5.4.

The last two arrangements serve as counterexamples to the following Main Theorem 2.6

Theorem 2.6 (Main Theorem). For the arrangements $\{1\},\{6\}$, and $\{7\}$ that each have a $\mathbb{Z}_{2}$ subgroup of their automorphism group, the map $\varphi: x \mapsto y, y \mapsto x$, and $z \mapsto z$ is a homeomorphism between the complements of representatives of the two components of the moduli space. 


\begin{tabular}{|c|c|c|c|c|}
\hline Case by section & Result & Over & $\operatorname{Aut}(\mathcal{A})$ & References \\
\hline$\{1\}: \operatorname{Eqn}(1)$ & Thm 4.3 & $\mathbb{R}$ & $\mathbb{Z}_{2}$ & From [ATY13]. See also [Zar13]. \\
\hline$\{6\}:\left(9_{3}\right)$.iii.ACG & Thm $\overline{\overline{4.5}}$ & $\mathbb{R}$ & $\mathbb{Z}_{2}$ & From [ACTY13. \\
\hline$\{7\}:\left(9_{3}\right)$.iii.BDF. & Thm 4.7 & $\mathbb{R}$ & $S_{4}$ & From [ACTY13. \\
\hline MacLane & Thm 5.1 & $\mathbb{C}$ & $\mathrm{GL}\left(2 ; \mathbb{F}_{3}\right)$ & From [Mac36]. See also [NY12, Example 4.3]. \\
\hline Nazir-Yoshinaga & Thm $\overline{5.3}$ & $\mathbb{C}$ & $S_{3}$ & From [NY12, Example 5.3]. \\
\hline 11.B.3.b.2.iii. & Thm $\overline{5.6}$ & $\mathbb{C}$ & $\mathbb{Z}_{2}$ & From [ACTY13. \\
\hline 11.B.3.b.2.iv. & Thm $\overline{5.8}$ & $\mathbb{C}$ & $\mathbb{Z}_{2}$ & From [ACTY13]. \\
\hline 11.B.2.iv. & Thm 5.10 & $\mathbb{C}$ & $\mathbb{Z}_{2}$ & From [ACTY13]. \\
\hline Falk-Sturmfels & Rem 6.2 & $\mathbb{R}$ & $\mathbb{Z}_{4}$ & From [CS97. See also [NY12, Example 5.2]. \\
\hline Rybnikov & Rem $\overline{6.3}$ & $\mathbb{C}$ & $S_{3} \times \mathbb{Z}_{2}$ & From [Ryb11]. See also [ABCRCAMB05. \\
\hline
\end{tabular}

TABLE 1. A list of the arrangements considered below.

Proof. This result summarizes results occuring later in the paper. For details see the proofs of Theorems 4.3, 4.5, and 4.7.

Question 2.7. Might this Main Theorem 2.6 hold in general under some suitable conditions?

\section{Geometric symmetry implies Combinatorial Symmetry}

Before we turn to demonstrating our algorithm, we prove the following elementary observation (implicitly mentioned in ABCRCAMB05]):

Proposition 3.1. Let $\mathcal{A}=\left\{L_{1}, \ldots, L_{n}\right\}$ and $\mathcal{A}^{\prime}=\left\{L_{1}^{\prime}, \ldots, L_{n}^{\prime}\right\}$ be two line arrangements in $\mathbb{C P}^{2}$ that represent different elements in the moduli space $\mathcal{M}_{\mathcal{A}}$ and let $\varphi: \mathbb{C P}^{2} \rightarrow \mathbb{C P}^{2}$ be the above reflection. If $\varphi\left(\cup_{i=1}^{n} L_{i}\right)=\cup_{i=1}^{n} L_{i}^{\prime}$ then there exists a lattice isomorphism $\sigma$ such that $\sigma \neq i d$ and $\sigma(\operatorname{Lat}(\mathcal{A}))=\operatorname{Lat}\left(\mathcal{A}^{\prime}\right)$.

Proof. Consider the arrangement $\mathcal{A}$ and it's image under the reflection $\varphi(\mathcal{A})=\left\{\varphi\left(L_{1}\right), \ldots, \varphi\left(L_{n}\right)\right\}$. The affine picture is shown in Figure 1 below. Since the moduli space is defined by modding out the action of $P G L(3, \mathbb{C})$, the arrangements $\mathcal{A}$ and $\varphi(\mathcal{A})$ represent the same element in $\mathcal{M}_{\mathcal{A}}$.

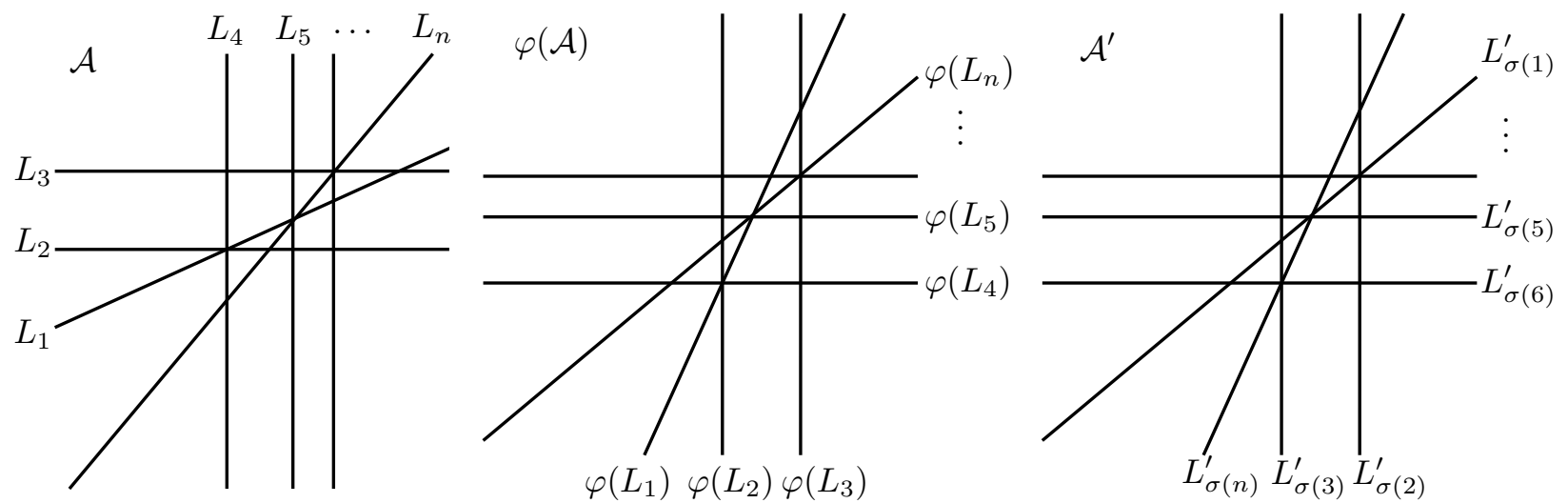

FiguRE 1. An arrangement $\mathcal{A}$, its image $\varphi(\mathcal{A})$ under the reflection, and the combinatorially equivalent arrangement $\mathcal{A}^{\prime}$.

We know that the union of the lines in $\varphi(\mathcal{A})$ and $\mathcal{A}^{\prime}$ produces the same curve in $\mathbb{C}^{2}$ :

$$
\cup_{i=1}^{n} \varphi\left(L_{i}\right)=\cup_{i=1}^{n} L_{i}^{\prime} .
$$


On the other hand, since the arrangements $\mathcal{A}$ and $\mathcal{A}^{\prime}$ represent different elements in $\mathcal{M}_{\mathcal{A}}$, we know also that as arrangements, $\varphi(\mathcal{A})$ and $\mathcal{A}^{\prime}$ are not equal. That is:

$$
\varphi\left(L_{i}\right) \neq L_{i}^{\prime} \quad \text { for some } i \in\{1, \ldots, n\} .
$$

Equations 3.1 and 3.2 imply that the arrangements $\varphi(\mathcal{A})$ and $\mathcal{A}^{\prime}$ must differ by the indices on their lines. This means that there exists a permutation $\sigma \neq i d$ of the index set $\{1, \ldots, n\}$ such that:

$$
\varphi\left(L_{i}\right)=L_{\sigma(i)}^{\prime} \quad \text { for all } \quad i \in\{1, \ldots, n\} .
$$

Moreover, since $\varphi(\mathcal{A})$ and $\mathcal{A}^{\prime}$ represent elements of the same moduli space $\mathcal{M}_{\mathcal{A}}$, we know that $\sigma$ is a lattice isomorphism of $\operatorname{Lat}(\mathcal{A})$.

Corollary 3.2. If $\operatorname{Aut}(\mathcal{A})$ is trivial, then disconnected components of $\mathcal{M}_{\mathcal{A}}$ are not symmetric to each other via a reflection in projective line in $\mathbb{C P}^{2}$.

Proof. Suppose by contrapositive there is a reflection in some line $L$. By changing coordinates in $\mathbb{C P}^{2}$, we can assume that the line $L$ has the defining equation $y=x$. The conclusion then follows directly from proposition 3.1 .

Corollary 3.2 justifies the approach taken in our algorithm. In order to find geometric reflections we must first identify combinatorial symmetries via $\operatorname{Aut}(\mathcal{A})$. What our examples show, furthermore, is that geometric reflections directly correspond to combinatorial reflections.

\section{Applichtion to Real ten-Line arrangements}

Although seven real cases of ten-line arrangements with disconnected moduli space were produced in [ATY13] and ACTY13, we only apply our algorithm to those that have a $\mathbb{Z}_{2}$ subgroup of the automorphism group. We start treating each of the cases $\{1\},\{6\}$ and $\{7\}$ by producing such a subgroup. We intentionally omit the proofs that the other four cases $\{2\}$ through $\{5\}$ do not contain such a symmetry.

Notation 4.1. We refer to $\{1\},\{6\}$ and $\{7\}$ as arrangements, so as to consider them as possible realizations of the combinatorics corresponding to these cases. Representatives of the two disconnected components of the moduli spaces of $\{i\}$ are denoted by $\{i\}^{+}$and $\{i\}^{-}$for $i=1,6,7$. The figures depicting these arrangements show real sections of affine arrangements obtained by choosing the line $z=0$ as the line at infinity.

Further treatment of Arrangement $\{1\}$ can be found in the last section of the Master's thesis of Zarkh [Zar13].

Example: Arrangement $\{1\}$. We consider the combinatorics of arrangement $\{1\}$ given by the configuration table in Table 2 .

\begin{tabular}{|ccc|ccc|ccc|c|}
\hline$L_{1}$ & $L_{2}$ & $L_{3}$ & $L_{4}$ & $L_{5}$ & $L_{6}$ & $L_{7}$ & $L_{8}$ & $L_{9}$ & $L_{10}$ \\
\hline$q_{1}$ & $q_{1}$ & $q_{1}$ & $q_{2}$ & $q_{2}$ & $q_{2}$ & $e_{1}$ & $e_{1}$ & $e_{2}$ & $e_{1}$ \\
$e_{7}$ & $e_{4}$ & $e_{2}$ & $e_{2}$ & $e_{3}$ & $e_{4}$ & $e_{3}$ & $e_{5}$ & $e_{3}$ & $q_{1}$ \\
$e_{8}$ & $e_{5}$ & $e_{6}$ & $e_{8}$ & $e_{7}$ & $e_{6}$ & $e_{4}$ & $e_{6}$ & $e_{5}$ & $q_{2}$ \\
& & & & & & $e_{8}$ & $e_{7}$ & & \\
\hline
\end{tabular}

TABLE 2. A configuration table for the triples and quadruples of the arrangement $\{1\}$.

Figure 2 shows an affine picture of a ten line arrangement realizing this configuration, with the line $L_{10}$ is plotted as the line at infinity.

Proposition 4.2. For the case $\{1\}$ from [ATY13, the group of symmetries Aut $(\{1\})$ is $\mathbb{Z}_{2}$. 


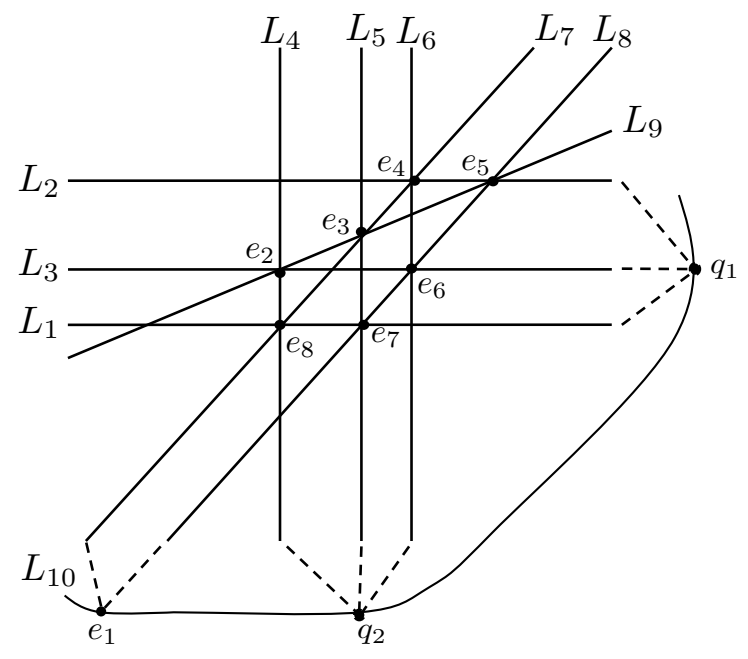

FiguRE 2. An arrangement realizing the combinatorics of $\{1\}$.

Proof. Let $\tau \in \operatorname{Aut}(\{1\})$. We determine $\tau$ using the following arguments:

$L_{10}$ is the only line that passes through two quadruples.

$$
\Longrightarrow \quad \tau\left(L_{10}\right)=L_{10}
$$

$L_{9}$ is the only line that passes through three triples and does not pass $\Longrightarrow \quad \tau\left(L_{9}\right)=L_{9}$ through a quadruple.

$e_{2}$ is the only triple on $L_{9}$ that is on two lines $\left(L_{3}\right.$ and $\left.L_{4}\right)$ that both $\Longrightarrow \quad \tau\left(e_{2}\right)=e_{2}$ pass through a quadruple.

$L_{7}$ and $L_{8}$ are the only lines that pass $\quad \Longrightarrow$ through four triples.

$$
\begin{array}{|l|}
\tau\left(L_{7}\right)=L_{7} \\
\hline \tau\left(L_{8}\right)=L_{8} \\
\hline
\end{array}
$$
or \begin{tabular}{|l|}
$\tau\left(L_{7}\right)=L_{8}$ \\
\hline$\tau\left(L_{8}\right)=L_{7}$ \\
\hline
\end{tabular}

$e_{3}$ and $e_{5}$ are intersection points of lines $\quad \Longrightarrow$ already determined.

$$
\begin{array}{|l|}
\tau\left(e_{3}\right)=e_{3} \\
\hline \tau\left(e_{5}\right)=e_{5} \\
\hline
\end{array}
$$$$
\begin{array}{|l|}
\tau\left(e_{3}\right)=e_{5} \\
\hline \tau\left(e_{5}\right)=e_{3} \\
\hline
\end{array}
$$

$L_{5}$ and $L_{2}$ are the last lines that pass

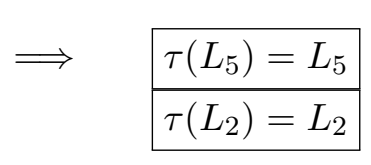$$
\begin{aligned}
& \tau\left(L_{5}\right)=L_{2} \\
& \hline \tau\left(L_{2}\right)=L_{5} \\
& \hline
\end{aligned}
$$

$L_{2}$ and $L_{5}$ lie on different quadruple points. $\quad \Longrightarrow$

$$
\begin{aligned}
& \tau\left(q_{1}\right)=q_{1} \\
& \tau\left(q_{2}\right)=q_{2} \\
& \hline
\end{aligned}
$$$$
\begin{array}{|l|}
\tau\left(q_{1}\right)=q_{2} \\
\hline \tau\left(q_{2}\right)=q_{1} \\
\hline
\end{array}
$$

$L_{3}$ and $L_{4}$ both pass through the fixed $e_{2} . \quad \Longrightarrow$

$$
\begin{array}{|l|}
\tau\left(L_{3}\right)=L_{3} \\
\hline \tau\left(L_{4}\right)=L_{4} \\
\hline
\end{array}
$$$$
\begin{array}{|l|}
\hline \tau\left(L_{3}\right)=L_{4} \\
\hline \tau\left(L_{4}\right)=L_{3} \\
\hline
\end{array}
$$ 
\begin{tabular}{ll|l|}
$\begin{array}{l}L_{1} \text { and } L_{6} \text { are mapped by which quadruple } \Longrightarrow \quad \begin{array}{l|}\tau\left(L_{1}\right)=L_{1} \\
\text { they are on. }\end{array} \\
\tau\left(L_{6}\right)=L_{6}\end{array}$ \\
\hline$\tau\left(L_{6}\right)=L_{1}$ \\
\hline
\end{tabular}

The above arguments imply that $\tau$ can be either the identity or $\sigma=\left(L_{1} L_{6}\right)\left(L_{2} L_{5}\right)\left(L_{3} L_{4}\right)\left(L_{7} L_{8}\right)$. Thus the automorphism group is $\mathbb{Z}_{2}$ with the above $\sigma$ as the non-trivial element.

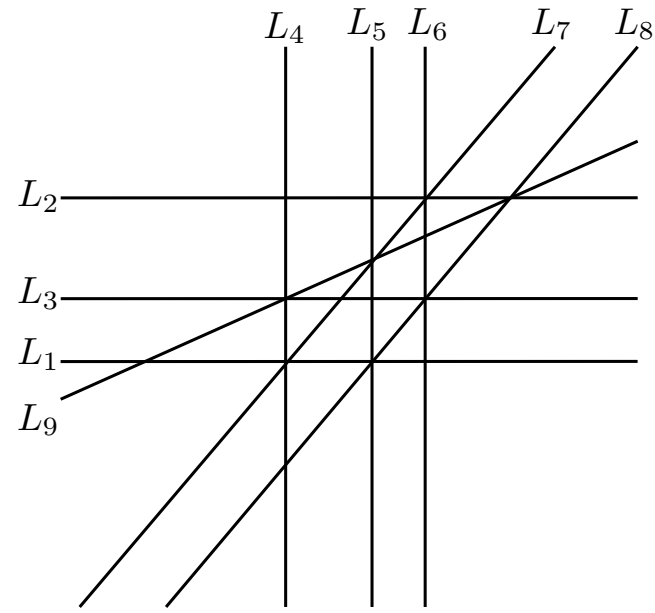

$\{1\}^{+}$

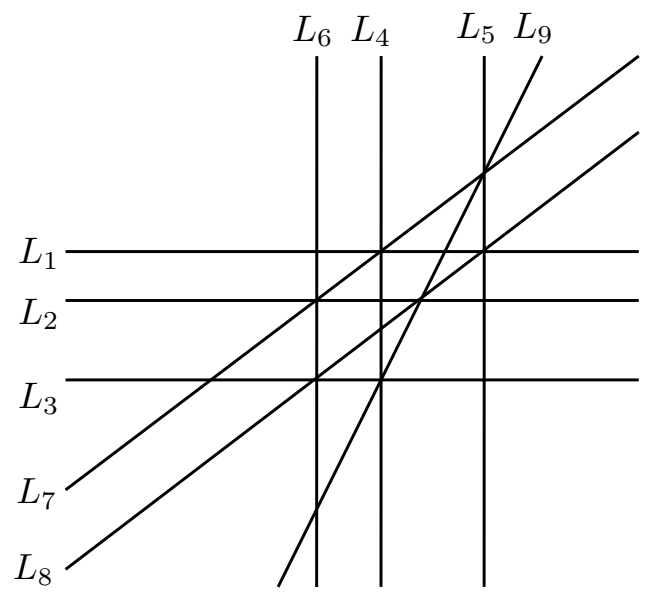

$\{1\}^{-}$

FIgURE 3. Arrangements in disconnected components of the moduli space $\mathcal{M}_{\{1\}}$.

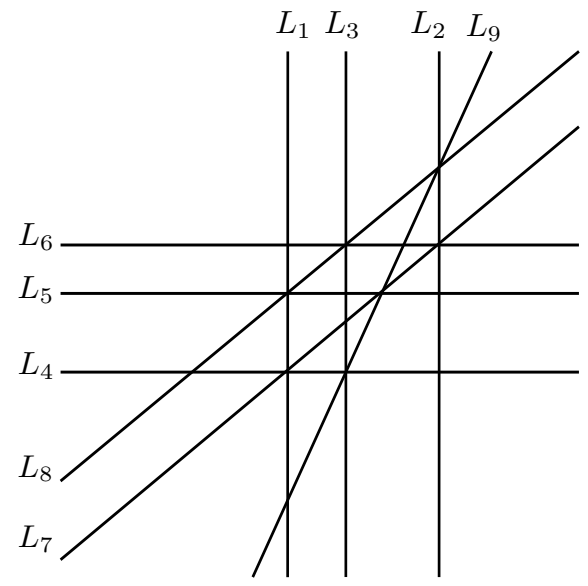

FiguRE 4. The reflected arrangement $\varphi\left(\{1\}^{+}\right)$.

Theorem 4.3. The homeomorphism $\varphi$ maps the pair $\left(\mathbb{C P}^{2},\{1\}^{+}\right)$to the pair $\left(\mathbb{C P}^{2},\{1\}^{-}\right)$.

Proof. We apply Algorithm 2.4:

(1) Set $\sigma=\left(L_{1} L_{6}\right)\left(L_{2} L_{5}\right)\left(L_{3} L_{4}\right)\left(L_{7} L_{8}\right)\left(L_{9}\right)\left(L_{10}\right)$.

(2) Set $L_{4}, L_{5}, L_{3}$, and $L_{2}$ as $x=0, x=z, y=0$, and $y=z$, respectively.

(3) This produces the following equations:

$$
\begin{gathered}
L_{1}: y=-t^{-1} z, \quad L_{2}: y=z, \quad L_{3}: y=0, \quad L_{4}: x=0, \quad L_{5}: x=z, \quad L_{6}: x=t z \\
L_{7}: y=x-t^{-1} z, \quad L_{8}: y=x-t z, \quad L_{9}: y=\left(1-t^{-1}\right) x, \quad L_{10}: z=0 .
\end{gathered}
$$


where $t^{ \pm}:=\frac{1 \pm \sqrt{5}}{2}$. Denote by $L_{i}^{ \pm}$the lines obtained from the above equations by setting $t=t^{ \pm}$. Then $\{1\}^{+}=\left\{L_{1}^{+}, \ldots, L_{10}^{+}\right\}$and $\{1\}^{-}=\left\{L_{1}^{-}, \ldots, L_{10}^{-}\right\}$.

Figure 3 displays the real section of the restriction of $\{1\}^{+}$and $\{1\}^{-}$to an affine chart obtained by choosing $L_{10}$, the line that passes through the two quadruples in both arrangements, to be the line $z=0$ at infinity.

(4) Clearly the depicted real graphs are symmetric and can be obtained from one another by applying the reflection $\varphi$. Moreover, switching $x$ and $y$ in the defining equations proves that $\varphi\left(L_{i}^{+}\right)=L_{\sigma(i)}^{-}$which shows that the reflection $\varphi$ corresponds to the combinatorial symmetry $\sigma$, where $t^{+} \mapsto t^{-}$because $\left(t^{+}\right)\left(t^{-}\right)=-1$. See Figures 3 and 4 for a geometric check.

Example: Arrangement $\{6\}$. In Table 3 we show the configuration table for the triples as given in ACTY13.

\begin{tabular}{|ccc|ccc|ccc|c|}
\hline$L_{1}$ & $L_{2}$ & $L_{3}$ & $L_{4}$ & $L_{5}$ & $L_{6}$ & $L_{7}$ & $L_{8}$ & $L_{9}$ & $L_{10}$ \\
\hline$e_{1}$ & $e_{1}$ & $e_{1}$ & $e_{8}$ & $e_{8}$ & $e_{8}$ & $e_{9}$ & $e_{9}$ & $e_{9}$ & $A$ \\
$e_{2}$ & $e_{4}$ & $e_{6}$ & $e_{2}$ & $e_{5}$ & $e_{3}$ & $e_{2}$ & $e_{4}$ & $e_{3}$ & $C$ \\
$e_{3}$ & $e_{5}$ & $e_{7}$ & $e_{4}$ & $e_{6}$ & $e_{7}$ & $e_{5}$ & $e_{7}$ & $e_{6}$ & $G$ \\
$G$ & & $C$ & $A$ & $G$ & & $C$ & & $A$ & \\
\hline
\end{tabular}

TABLE 3. A configuration table for the triples of the arrangement $\{6\}$.

Proposition 4.4. For the case $\{6\}$ from ACTY13, the group of symmetries Aut $(\{6\})$ is $\mathbb{Z}_{2}$.

Proof. Any symmetry of the arrangement must respect the lines that contain exactly three triples, and thus it must fix $\left\{L_{2}, L_{6}, L_{8}, L_{10}\right\}$ setwise. There are two common triples $e_{4}, e_{7}$ amongst these lines, and so the line $L_{8}$ which contains both of these and the line $L_{10}$ which contains neither of these must both be fixed. This also fixes the triple $e_{9}$ on the line $L_{8}$.

Aside from the identity on this set, this also gives the transposition $\left(e_{4} e_{7}\right)\left(L_{2} L_{6}\right)$, which must transpose the sets $\left\{e_{1}, e_{5}\right\}$ and $\left\{e_{3}, e_{8}\right\}$. By the triples $e_{4}, e_{7}$ on two other lines, this transposition also gives $\left(L_{4} L_{3}\right)$, which must transpose the sets $\left\{e_{8}, e_{2}, e_{A}\right\}$ and $\left\{e_{1}, e_{6}, e_{C}\right\}$. This confirms that the transposition must include both $\left(e_{1} e_{8}\right)$ and $\left(e_{A} e_{C}\right)$, the latter since the line $L_{10}$ is fixed. This of course fixes $e_{G}$ and must include the transpositions $\left(e_{2} e_{6}\right)$ and $\left(e_{3} e_{5}\right)$.

Thus the transposition gives $\left(L_{1} L_{5}\right)\left(L_{2} L_{6}\right)\left(L_{3} L_{4}\right)\left(L_{7} L_{9}\right)$ while fixing the lines $L_{8}$ and $L_{10}$.

Lastly we consider the pointwise identity on the set $\left\{L_{2}, L_{6}, L_{8}, L_{10}\right\}$ from above. This fixes the triples $e_{4}, e_{7}$, which also fixes $e_{1}, e_{8}$ followed by $e_{A}, e_{C}$ and $e_{2}, e_{6}$ and $e_{3}, e_{5}$ according to the same argument used above for the transposition.

This gives the identity, and thus there are no other symmetries.

Theorem 4.5. The homeomorphism $\varphi$ maps the pair $\left(\mathbb{C P}^{2},\{6\}^{+}\right)$to the pair $\left(\mathbb{C P}^{2},\{6\}^{-}\right)$.

Proof. We apply Algorithm 2.4.

(1) Set $\sigma=\left(L_{1} L_{5}\right)\left(L_{2} L_{6}\right)\left(L_{3} L_{4}\right)\left(L_{7} L_{9}\right)\left(L_{8}\right)\left(L_{10}\right)$.

(2) Set $L_{4}, L_{6}, L_{3}$, and $L_{2}$ as $x=0, x=z, y=0$, and $y=z$, respectively.

(3) This produces the following equations:

$$
\begin{gathered}
L_{1}: y=-t^{-1} z, \quad L_{2}: y=z, \quad L_{3}: y=0, \quad L_{4}: x=0, \quad L_{5}: x=t z, \quad L_{6}: x=z, \\
L_{7}: y=\frac{1+t^{-1}}{t} x-t^{-1} z, \quad L_{8}: y=-x+z, \quad L_{9}: y=\frac{t^{-1}}{t-1}(x-t z), \quad L_{10}: y=\frac{1+t^{-1}}{t-1} x-\frac{1}{t-1},
\end{gathered}
$$

where $t^{2}+t-1=0$, or $t^{ \pm}=\frac{-1 \pm \sqrt{5}}{2}$. Denote by $L_{i}^{ \pm}$the lines obtained by setting $t=t^{ \pm}$. Then $\{1\}^{+}=\left\{L_{1}^{+}, \ldots, L_{10}^{+}\right\}$and $\{1\}^{-}=\left\{L_{1}^{-}, \ldots, L_{10}^{-}\right\}$. 
(4) It is easy to check that $\varphi\left(L_{i}^{+}\right)=L_{\sigma(i)}^{-}$, where $t^{+} \mapsto t^{-}$because $\left(t^{+}\right)\left(t^{-}\right)=-1$. See Figures 5 and 6 for a geometric check.

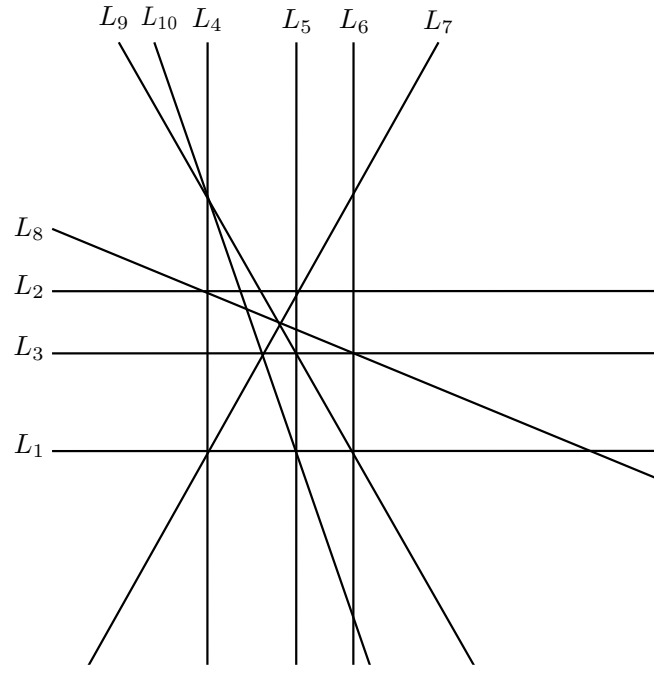

$\{6\}^{+}$

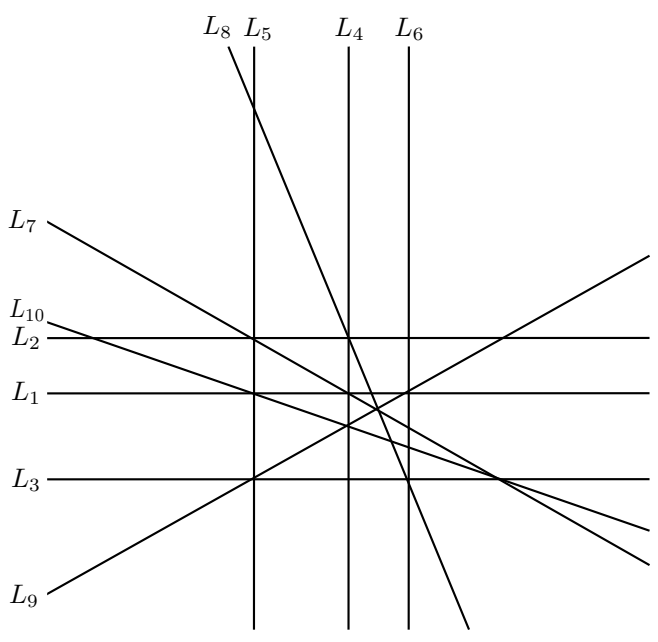

$\{6\}^{-}$

FiguRE 5. Arrangements in disconnected components of the moduli space $\mathcal{M}_{\{6\}}$.

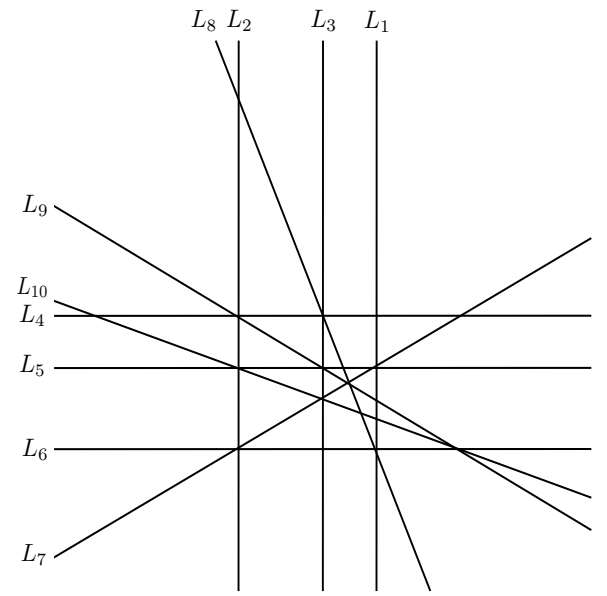

FiguRE 6. The reflected arrangement $\varphi\left(\{6\}^{+}\right)$.

Example: Arrangement $\{7\}$. In Table 4 we show the configuration table for the triples as given in ACTY13.

Proposition 4.6. For the case $\{7\}$ from [ACTY13], the group of symmetries Aut $(\{7\})$ is $S_{4}$.

Proof. First observe that there are exactly four lines $L_{1}, L_{5}, L_{8}, L_{10}$ that contain exactly three triples. Then observe that no pair of these lines contains a common triple. Thus we may consider symmetries that are subsets of $S_{4}$ that preserve these lines.

We refer to a graphical interpretation of $\{7\}$ in Figure 7 with six lines as its vertices and twelve triples as its edges. This graph can be reflected and rotated to show $S_{3}$ symmetry while fixing the line $L_{10}$ (which contains the elements $B, D, F$ bolded in the figure). One can see both the reflection 


\begin{tabular}{|ccc|ccc|ccc|c|}
\hline$L_{1}$ & $L_{2}$ & $L_{3}$ & $L_{4}$ & $L_{5}$ & $L_{6}$ & $L_{7}$ & $L_{8}$ & $L_{9}$ & $L_{10}$ \\
\hline$e_{1}$ & $e_{1}$ & $e_{1}$ & $e_{8}$ & $e_{8}$ & $e_{8}$ & $e_{9}$ & $e_{9}$ & $e_{9}$ & $B$ \\
$e_{2}$ & $e_{4}$ & $e_{6}$ & $e_{2}$ & $e_{5}$ & $e_{3}$ & $e_{2}$ & $e_{4}$ & $e_{3}$ & $D$ \\
$e_{3}$ & $e_{5}$ & $e_{7}$ & $e_{4}$ & $e_{6}$ & $e_{7}$ & $e_{5}$ & $e_{7}$ & $e_{6}$ & $F$ \\
& $F$ & $B$ & $B$ & & $D$ & $D$ & & $F$ & \\
\hline
\end{tabular}

TABLE 4. A configuration table for the triples of the arrangement $\{7\}$.

(through a vertical line through 1 and $D)$ giving $(B F)$ and $\left(L_{5} L_{8}\right)\left(L_{2} L_{3}\right)\left(L_{4} L_{9}\right)\left(L_{6} L_{7}\right)$ as well as the cyclic rotation $(B D F)$ and $\left(\begin{array}{llll}L_{2} & L_{4} & L_{7}\end{array}\right)\left(\begin{array}{lll}L_{3} & L_{6} & L_{9}\end{array}\right)$.

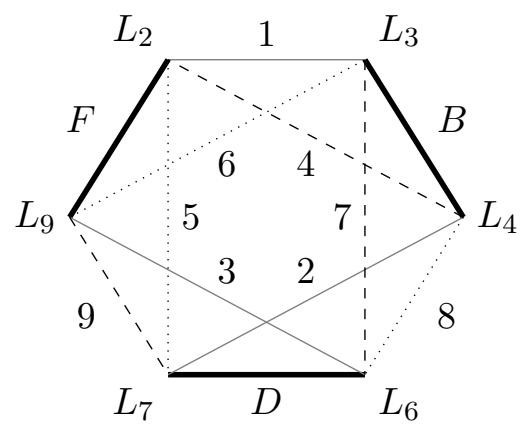

FIgURE 7. A depiction of $\{7\}$ showing its symmetries: the graph has six lines as its vertices and twelve triples as its edges. Four lines are not shown: the solid triples lie on $L_{1}$, the dotted triples lie on $L_{5}$, the dashed triples lie on $L_{8}$, and the bolded triples lie on $L_{10}$.

Furthermore, because the three edges missing from the complete graph $K_{6}$ on six vertices correspond to doubles, $L_{2} \cap L_{6}, L_{3} \cap L_{7}$, and $L_{4} \cap L_{9}$, and not triples, there can be no additional symmetries with the line $L_{10}$ fixed.

In order to produce $S_{4}$ we have left to produce a transposition taking $L_{10}$ to one of the other three lines, say $L_{8}$. One can take the symmetry $\left(L_{2} L_{6}\right)\left(L_{3} L_{9}\right)\left(L_{4} L_{7}\right)\left(L_{8} L_{10}\right)$ fixing the lines $L_{1}$ and $L_{5}$.

Now to see that there are no other symmetries, we fix each of the four lines $L_{1}, L_{5}, L_{8}$, and $L_{10}$ and consider where the triple $e_{1}$ might be sent amongst the triples $e_{1}, e_{2}$, and $e_{3}$. We consider the remaining four lines containing either the triple $e_{1}$ or its image. If $e_{1}$ is not sent to itself, then in both cases the triples on one of the original four lines behave with $\mathbb{Z}_{3}$ symmetry while the triples on the other three of the original four lines behave with $\mathbb{Z}_{2}$ symmetry: this results in a contradiction for the two final lines. If the triple $e_{1}$ is sent to itself, then the $\mathbb{Z}_{2}$ symmetries of the triples on the four original lines are not compatible with each other, also leading to a contradiction.

Theorem 4.7. The homeomorphism $\varphi$ maps the pair $\left(\mathbb{C P}^{2},\{7\}^{+}\right)$to the pair $\left(\mathbb{C P}^{2},\{7\}^{-}\right)$.

Proof. We apply Algorithm 2.4:

(1) Set $\sigma=\left(L_{1} L_{5}\right)\left(L_{2} L_{6}\right)\left(L_{3} L_{4}\right)\left(L_{7} L_{9}\right)\left(L_{8}\right)\left(L_{10}\right)$.

(2) Set $L_{4}, L_{5}, L_{3}$, and $L_{1}$ as $x=0, x=z, y=0$, and $y=z$, respectively.

(3) This produces the following equations:

$$
\begin{gathered}
L_{1}: y=z, \quad L_{2}: y=-t^{-1} z, \quad L_{3}: y=0, \quad L_{4}: x=0, \quad L_{5}: x=z, \quad L_{6}: x=t z \\
L_{7}: y=-t x+z, \quad L_{8}: y=t^{-2} x-t^{-1} z, \quad L_{9}: y=t(x-z), \quad L_{10}: y=-x
\end{gathered}
$$


where $t^{2}-t-1=0$, or $t^{ \pm}=\frac{1 \pm \sqrt{5}}{2}=\left(-t^{\mp}\right)^{-1}$. Denote by $L_{i}^{ \pm}$the lines obtained by setting $t=t^{ \pm}$. Then $\{1\}^{+}=\left\{L_{1}^{+}, \ldots, L_{10}^{+}\right\}$and $\{1\}^{-}=\left\{L_{1}^{-}, \ldots, L_{10}^{-}\right\}$.

(4) It is easy to check that $\varphi\left(L_{i}^{+}\right)=L_{\sigma(i)}^{-}$, where $t^{+} \mapsto t^{-}$because $\left(t^{+}\right)\left(t^{-}\right)=-1$. See Figures 8 and 9 for a geometric check.

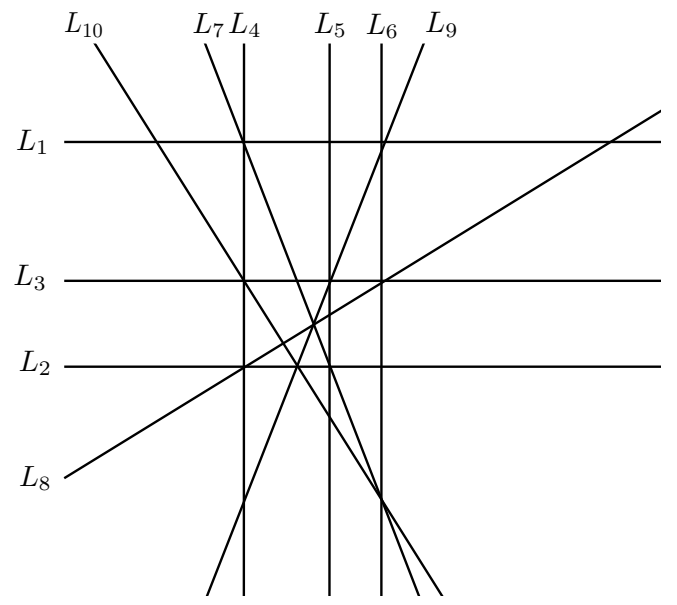

$\{7\}^{+}$

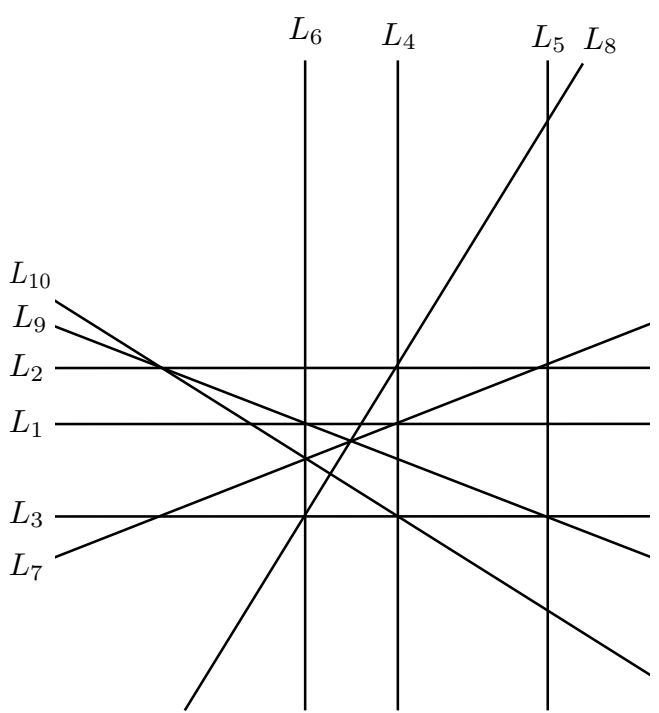

$\{7\}^{-}$

Figure 8. Arrangements in disconnected components of the moduli space $\mathcal{M}_{\{7\}}$.

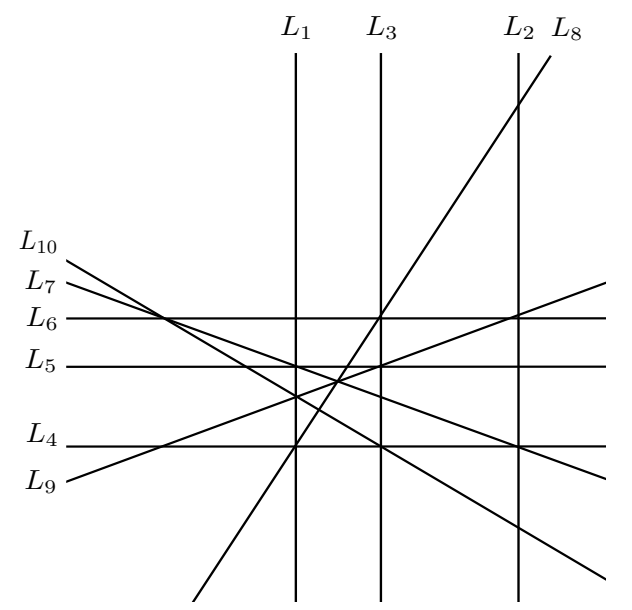

FiguRE 9. The reflected arrangement $\varphi\left(\{7\}^{+}\right)$.

\section{Application to COMPlex arrangements that are AlREady not Zariski pairs}

To show the robustness of the algorithm above, we now consider examples that were already found to be complex conjugate and thus not Zariski pairs.

The first subsection considers arrangements from the literature that have this $\mathbb{Z}_{2}$ symmetry as a subgroup of the automorphism group. In the second subsection we consider the three arrangements that contain a $\mathbb{Z}_{2}$ subgroup out of the five complex conjugate arrangements that were produced in ACTY13. 
5.1. Application to complex arrangements appearing in the literature. We consider the MacLane arrangement [Mac36] of eight lines and the Nazir-Yoshinaga [NY12] arrangement of nine lines. We apply our technique to these examples and show that there is some $\mathbb{Z}_{2}$ symmetry subgroup which yields an obvious reflection.

Example: MacLane arrangement. We start with the combinatorics of the arrangement obtained from the geometry in Example 4.3 of [NY12]. We give a configuration table for the triples in Table 5 .

\begin{tabular}{|ccc|ccc|cc|}
\hline$H_{1}$ & $H_{2}$ & $H_{3}$ & $H_{4}$ & $H_{5}$ & $H_{6}$ & $H_{7}$ & $H_{8}$ \\
\hline$e_{1}$ & $e_{1}$ & $e_{1}$ & $e_{8}$ & $e_{8}$ & $e_{8}$ & $e_{2}$ & $e_{3}$ \\
$e_{2}$ & $e_{4}$ & $e_{6}$ & $e_{2}$ & $e_{5}$ & $e_{3}$ & $e_{5}$ & $e_{4}$ \\
$e_{3}$ & $e_{5}$ & $e_{7}$ & $e_{4}$ & $e_{6}$ & $e_{7}$ & $e_{7}$ & $e_{6}$ \\
\hline
\end{tabular}

TABLE 5. A configuration table for the triples of the MacLane arrangement.

As stated in Example 2.3 of [ABCRCAMB05, the group of symmetries for the MacLane arrangement is $\mathrm{GL}\left(2 ; \mathbb{F}_{3}\right)$.

Theorem 5.1. The homeomorphism $\widetilde{\varphi}: x \mapsto \bar{y}, y \mapsto \bar{x}$, and $z \mapsto \bar{z}$ maps the pair $\left(\mathbb{C P}^{2},+\right)$ to the pair $\left(\mathbb{C P}^{2},-\right)$.

Proof. We apply Algorithm 2.4:

(1) Set $\sigma=\left(H_{1} H_{5}\right)\left(H_{2} H_{6}\right)\left(H_{3} H_{4}\right)\left(H_{7} H_{8}\right)$.

(2) Set $H_{1}, H_{2}, H_{5}$, and $H_{6}$ as $x=0, x=z, y=0$, and $y=z$, respectively.

(3) This produces the following equations:

$$
\begin{gathered}
H_{1}: x=0, \quad H_{2}: x=z, \quad H_{3}: x=t z, \quad H_{4}: y=t z \\
H_{5}: y=0, \quad H_{6}: y=z, \quad H_{7}: y=-t x+t z, \quad H_{8}: y=-t^{-1} x+z,
\end{gathered}
$$

where $t^{2}-t+1=0$, or $t^{ \pm}=\frac{1 \pm \sqrt{3} i}{2}$.

(4) It is easy to check that $\varphi\left(L_{i}^{+}\right)=L_{\sigma(i)}^{-}$, where $t^{+} \mapsto t^{-}$with $\left(t^{+}\right)\left(t^{-}\right)=1$.

Example: Nazir-Yoshinaga arrangement. We start with the combinatorics of the arrangement obtained from the geometry in Example 4.3 of [NY12]. We give a configuration table for the triples in Table 6 .

\begin{tabular}{|ccc|ccc|ccc|}
\hline$L_{1}$ & $L_{2}$ & $L_{3}$ & $L_{4}$ & $L_{5}$ & $L_{6}$ & $L_{7}$ & $L_{8}$ & $L_{9}$ \\
\hline$e_{1}$ & $e_{1}$ & $e_{1}$ & $e_{10}$ & $e_{10}$ & $e_{10}$ & $e_{2}$ & $e_{3}$ & $e_{4}$ \\
$e_{2}$ & $e_{5}$ & $e_{8}$ & $e_{2}$ & $e_{3}$ & $e_{4}$ & $e_{7}$ & $e_{5}$ & $e_{6}$ \\
$e_{3}$ & $e_{6}$ & $e_{9}$ & $e_{5}$ & $e_{6}$ & $e_{7}$ & $e_{9}$ & $e_{9}$ & $e_{8}$ \\
$e_{4}$ & $e_{7}$ & & $e_{8}$ & & & & & \\
\hline
\end{tabular}

TABLE 6. A configuration table for the triples of the Nazir-Yoshinaga arrangement.

Proposition 5.2. For the Nazir-Yoshinaga arrangement from [NY12], the group of symmetries is $S_{3}$ with a $\mathbb{Z}_{2}$ subgroup generated by $\sigma$.

Proof. Any symmetry of the arrangement must respect the lines that contain exactly four triples, and thus it must fix $\left\{L_{1}, L_{2}, L_{4}\right\}$ setwise. There are three common triples $e_{1}, e_{2}, e_{5}$ amongst these lines, each in a different pair. We show this gives the $S_{3}$ by producing a $\mathbb{Z}_{3}$ symmetry and the $\mathbb{Z}_{2}$ symmetry generated by $\sigma$. 
These three lines contain all triples but $e_{9}$, and so this triple must be fixed. Furthermore, the three lines $L_{3}, L_{7}, L_{8}$ through this triple each contain one of the three aforenamed triples $e_{1}, e_{2}, e_{5}$. Thus there is no more symmetry.

To obtain the $\mathbb{Z}_{3}$ symmetry we consider $\left(e_{1} e_{2} e_{5}\right)$ which gives $\left(L_{1} L_{4} L_{2}\right)\left(\begin{array}{lll}L_{3} & L_{7} & L_{8}\end{array}\right)$ and then $\left(e_{8} e_{7} e_{3}\right)$. This in turn gives $\left(e_{4} e_{10} e_{6}\right)$ and $\left(L_{5} L_{9} L_{6}\right)$.

There is also a transposition $\sigma$ fixing the triple $e_{1}$ and thus the line $L_{4}$ that gives $\left(e_{2} e_{5}\right)\left(L_{1} L_{2}\right)$. This gives $\left(L_{7} L_{8}\right)$ fixing the line $L_{3}$, which gives $\left(e_{3} e_{7}\right)$ fixing $e_{8}$. Finally this gives $\left(e_{4} e_{6}\right)\left(L_{5} L_{6}\right)$ fixing triples $e_{9}, e_{10}$ and the line $L_{9}$.

Theorem 5.3. The homeomorphism $\varphi$ maps the pair $\left(\mathbb{C P}^{2},+\right)$ to the pair $\left(\mathbb{C P}^{2},-\right)$.

Proof. We apply Algorithm 2.4

(1) Set $\sigma=\left(L_{1} L_{2}\right)\left(L_{3}\right)\left(L_{4}\right)\left(L_{5} L_{6}\right)\left(L_{7} L_{8}\right)\left(L_{9}\right)$.

(2) Set $L_{2}, L_{7}, L_{1}$, and $L_{8}$ as $x=0, x=z, y=0$, and $y=z$, respectively.

(3) This produces the following equations:

$$
\begin{gathered}
L_{1}: y=0, \quad L_{2}: x=0, \quad L_{3}: y=x, \quad L_{4}: y=-x+z, \quad L_{5}: y=\frac{1}{2 t} z, \\
L_{6}: x=t z, \quad L_{7}: x=z, \quad L_{8}: y=z, \quad L_{9}: y=2 t^{2} x+\frac{1}{2 t} z,
\end{gathered}
$$

where $2 t^{2}-2 t+1=0$, or $t^{ \pm}=\frac{1 \pm i}{2}$.

(4) It is easy to check that $\varphi\left(L_{i}^{+}\right)=L_{\sigma(i)}^{-}$, where $t^{+} \mapsto t^{-}$because $\left(t^{+}\right)\left(t^{-}\right)=\frac{1}{2}$.

5.2. Application to complex ten-line arrangements. To verify the algorithm above, we now consider examples that were already found to be complex conjugate and thus not Zariski pairs.

We consider the three complex cases 11.B.3.b.2.iii, 11.B.3.b.2.iv and 11.B.2.iv. The (complex) dimension of the moduli spaces of all these cases is one, and so there will be another variable to consider. Pay close attention to following usage of notation.

Notation 5.4. In the discussion below the variable $s$ will give us the two disconnected components, as a solution $s^{ \pm}$to some quadratic defining equation, while the variable $t$ will act as the free variable giving dimension one.

It is important to distinguish between, on the one hand, the merely two distinct allowable values $s^{ \pm}$of the first variable and, on the other hand, the full dimensional variable $t$ of each of the two disconnected components.

Even though it is a terrible abuse of notation and would lead to some confusion were it not for this note, when we restrict to the component given by $s^{+}$we will call the one-dimensional family of arrangements $t^{+}$. The same holds similarly for $t^{-}$.

Now just as we have the map $\varphi$ sending, say, $x$ to $y$, we will also need a map comparing values of $t$ in one component to values of $t$ in the other component.

However, instead of providing this map, we will simply choose one value of the $t$ in each component to show its symmetry. The rest of each component can be treated by Randell's Isotopy Theorem Ran89, as mentioned above.

Example: Arrangement 11.B.3.b.2.iii. In Table 7 we show the configuration table for the triples as given in ACTY13.

Proposition 5.5. For the case 11.B.3.b.2.iii. from [ACTY13, the group of symmetries is $\mathbb{Z}_{2}$.

Proof. Any symmetry of the arrangement must respect the lines that contain exactly four triples, and thus it must fix $\left\{L_{1}, L_{2}, L_{3}\right\}$ setwise. There are three common triples $e_{1}, e_{2}, e_{3}$ amongst these lines. Since the triples $e_{1}, e_{2}, e_{3}$ lie on three other lines, we know that the set $\left\{L_{4}, L_{5}, L_{6}\right\}$ must 


\begin{tabular}{|ccc|ccc|cccc|}
\hline$L_{1}$ & $L_{2}$ & $L_{3}$ & $L_{4}$ & $L_{5}$ & $L_{6}$ & $L_{7}$ & $L_{8}$ & $L_{9}$ & $L_{10}$ \\
\hline$e_{1}$ & $e_{1}$ & $e_{2}$ & $e_{1}$ & $e_{2}$ & $e_{3}$ & $e_{4}$ & $e_{5}$ & $e_{5}$ & $e_{7}$ \\
$e_{2}$ & $e_{3}$ & $e_{3}$ & $e_{10}$ & $e_{6}$ & $e_{4}$ & $e_{7}$ & $e_{6}$ & $e_{9}$ & $e_{9}$ \\
$e_{4}$ & $e_{6}$ & $e_{8}$ & $e_{11}$ & $e_{10}$ & $e_{11}$ & $e_{8}$ & $e_{8}$ & $e_{10}$ & $e_{11}$ \\
$e_{5}$ & $e_{7}$ & $e_{9}$ & & & & & & & \\
\hline
\end{tabular}

TABLE 7. A configuration table for the triples of the arrangement 11.B.3.b.2.iii.

also be fixed. There are two common triples $e_{10}, e_{11}$ amongst these three lines, and hence the line $L_{4}$ which contains both of these must be fixed.

Aside from the identity on the set $\left\{L_{4}, L_{5}, L_{6}\right\}$, this also gives the transposition $\left(e_{2} e_{3}\right)\left(e_{6} e_{4}\right)\left(L_{5} L_{6}\right)$. This transposition gives $\left(L_{1} L_{2}\right)$ and fixes the line $L_{3}$.

By the triples $e_{4}, e_{6}$ on two other lines, transposition $\left(e_{2} e_{3}\right)\left(e_{6} e_{4}\right)\left(L_{5} L_{6}\right)$ also gives $\left(L_{7} L_{8}\right)$, which must transpose the set $\left\{e_{5}, e_{7}\right\}$ and fix the triple $e_{8}$. Similarly, transposition $\left(e_{5} e_{7}\right)$ gives the transposition $\left(L_{9} L_{10}\right)$, which must fix the triple $e_{9}$.

Thus we have $\left(L_{1} L_{2}\right)\left(L_{5} L_{6}\right)\left(L_{7} L_{8}\right)\left(L_{9} L_{10}\right)$ while fixing the lines $L_{3}$ and $L_{4}$.

Lastly we consider the pointwise identity on the set $\left\{L_{4}, L_{5}, L_{6}\right\}$ from above. This fixes the triples $e_{1}, e_{2}, e_{3}$ which also fixes $e_{10}, e_{11}$ followed by $e_{4}, e_{6}$ and $e_{5}, e_{7}$ according to the same argument used above for the transposition. This gives the identity, and thus there are no other symmetries.

Theorem 5.6. The homeomorphism $\varphi$ maps the pair $\left(\mathbb{C P}^{2}, t^{+}\right)$to the pair $\left(\mathbb{C P}^{2}, t^{-}\right)$.

Proof. We apply Algorithm 2.4:

(1) Set $\sigma=\left(L_{1} L_{2}\right)\left(L_{3}\right)\left(L_{4}\right)\left(L_{5} L_{6}\right)\left(L_{7} L_{8}\right)\left(L_{9} L_{10}\right)$.

(2) Set $L_{1}, L_{5}, L_{2}$, and $L_{6}$ as $x=0, x=z, y=0$, and $y=z$, respectively.

(3) This produces the following equations:

$$
\begin{gathered}
L_{1}: x=0, \quad L_{2}: y=0, \quad L_{3}: z=0, \quad L_{4}: y=(s-t) x, \quad L_{5}: x=z, \quad L_{6}: y=z, \\
L_{7}: y=t x+z, \quad L_{8}: y=t x-t z, \quad L_{9}: y=s x-t z, \quad L_{10}: y=s x+\frac{s}{t} z,
\end{gathered}
$$

where $s^{2}-t s+t^{2}=0$, or $s^{ \pm}=\frac{1 \pm \sqrt{3} i}{2} t$.

(4) Setting $t=1$ we get $s^{2}-s+1=0$, or $s^{ \pm}=\frac{1 \pm \sqrt{3} i}{2}$. It is easy to check that $\varphi\left(L_{i}^{+}\right)=L_{\sigma(i)}^{-}$, where $s^{+} \mapsto s^{-}$because $\left(s^{+}\right)\left(s^{-}\right)=1$. We apply Randell's Isotopy Theorem to the rest of each component.

Example: Arrangement 11.B.3.b.2.iv. In Table 8 we show the configuration table for the triples as given in ACTY13.

\begin{tabular}{|ccc|ccc|cccc|}
\hline$L_{1}$ & $L_{2}$ & $L_{3}$ & $L_{4}$ & $L_{5}$ & $L_{6}$ & $L_{7}$ & $L_{8}$ & $L_{9}$ & $L_{10}$ \\
\hline$e_{1}$ & $e_{1}$ & $e_{2}$ & $e_{1}$ & $e_{2}$ & $e_{3}$ & $e_{4}$ & $e_{5}$ & $e_{5}$ & $e_{7}$ \\
$e_{2}$ & $e_{3}$ & $e_{3}$ & $e_{10}$ & $e_{6}$ & $e_{4}$ & $e_{7}$ & $e_{6}$ & $e_{9}$ & $e_{9}$ \\
$e_{4}$ & $e_{6}$ & $e_{8}$ & $e_{11}$ & $e_{11}$ & $e_{10}$ & $e_{8}$ & $e_{8}$ & $e_{10}$ & $e_{11}$ \\
$e_{5}$ & $e_{7}$ & $e_{9}$ & & & & & & & \\
\hline
\end{tabular}

TABLE 8. A configuration table for the triples of the arrangement 11.B.3.b.2.iv.

Proposition 5.7. For the case 11.B.3.b.2.iv. from [ACTY13, the group of symmetries is $\mathbb{Z}_{2}$.

Proof. The proof is the same as the proof of Proposition 5.5. We omit the details.

Theorem 5.8. The homeomorphism $\varphi$ maps the pair $\left(\mathbb{C P}^{2}, t^{+}\right)$to the pair $\left(\mathbb{C P}^{2}, t^{-}\right)$. 
Proof. We apply Algorithm 2.4:

(1) Set $\sigma=\left(L_{1} L_{2}\right)\left(L_{3}\right)\left(L_{4}\right)\left(L_{5} L_{6}\right)\left(L_{7} L_{8}\right)\left(L_{9} L_{10}\right)$.

(2) Set $L_{1}, L_{5}, L_{2}$, and $L_{6}$ as $x=0, x=z, y=0$, and $y=z$, respectively.

(3) This produces the following equations:

$$
\begin{gathered}
L_{1}: x=0, \quad L_{2}: y=0, \quad L_{3}: z=0, \quad L_{4}: y=\frac{t}{1+s} x, \quad L_{5}: x=z, \quad L_{6}: y=z, \\
L_{7}: y=s x+z, \quad L_{8}: y=s x-s z, \quad L_{9}: y=t x-s z, \quad L_{10}: y=t x+\frac{t}{s} z,
\end{gathered}
$$

where $s^{2}+s+1=0$, or $s^{ \pm}=\frac{-1 \pm \sqrt{3} i}{2}$.

(4) We set $t=1$. It is easy to check that $\varphi\left(L_{i}^{+}\right)=L_{\sigma(i)}^{-}$, where $s^{+} \mapsto s^{-}$because $\left(s^{+}\right)\left(s^{-}\right)=1$. We apply Randell's Isotopy Theorem to the rest of each component.

Example: Arrangement 11.B.2.iv. In Table 9 we show the configuration table for the triples as given in ACTY13.

\begin{tabular}{|ccc|cc|cc|c|cc|}
\hline$L_{1}$ & $L_{2}$ & $L_{3}$ & $L_{4}$ & $L_{5}$ & $L_{6}$ & $L_{7}$ & $L_{8}$ & $L_{9}$ & $L_{10}$ \\
\hline$e_{1}$ & $e_{1}$ & $e_{2}$ & $e_{1}$ & $e_{2}$ & $e_{5}$ & $e_{6}$ & $e_{6}$ & $e_{7}$ & $e_{7}$ \\
$e_{2}$ & $e_{5}$ & $e_{8}$ & $e_{8}$ & $e_{5}$ & $e_{10}$ & $e_{10}$ & $e_{9}$ & $e_{9}$ & $e_{8}$ \\
$e_{3}$ & $e_{6}$ & $e_{9}$ & $e_{11}$ & $e_{11}$ & $e_{3}$ & $e_{4}$ & $e_{11}$ & $e_{4}$ & $e_{3}$ \\
$e_{4}$ & $e_{7}$ & $e_{10}$ & & & & & & & \\
\hline
\end{tabular}

TABLE 9. A configuration table for the triples of the arrangement 11.B.2.iv.

Proposition 5.9. For the case 11.B.2.iv. from [ACTY13], the group of symmetries is $\mathbb{Z}_{2}$.

Proof. Any symmetry of the arrangement must respect the lines that contain exactly four triples, and thus it must fix $\left\{L_{1}, L_{2}, L_{3}\right\}$ setwise. There are two common triples $e_{1}, e_{2}$ amongst these lines, and hence the line $L_{1}$ which contains both of these must be fixed.

Aside from the identity on this set, this also gives the transposition $\left(e_{1} e_{2}\right)\left(L_{2} L_{3}\right)$. By the triples $e_{1}, e_{2}$ on two other lines, this transposition also gives $\left(L_{4} L_{5}\right)$, which must transpose the set $\left\{e_{5}, e_{8}\right\}$ and fix $e_{11}$. Similarly, this gives the transposition $\left(L_{6} L_{10}\right)$, which must transpose the set $\left\{e_{7}, e_{10}\right\}$ and fix $e_{3}$. Thus we get the transposition $\left(L_{7} L_{9}\right)$, which must transpose the set $\left\{e_{6}, e_{9}\right\}$ and fix $e_{4}$. Therefore the line $L_{8}$ must be fixed. Thus we have $\left(L_{2} L_{3}\right)\left(L_{4} L_{5}\right)\left(L_{6} L_{10}\right)\left(L_{7} L_{9}\right)$ while fixing the lines $L_{1}$ and $L_{8}$.

Lastly we consider the pointwise identity on the set $\left\{L_{1}, L_{2}, L_{3}\right\}$ from above. This fixes the triples $e_{1}, e_{2}$, which also fixes $e_{5}, e_{8}$ followed by $e_{7}, e_{10}$ and $e_{6}, e_{9}$ according to the same argument used above for the transposition. This gives the identity, and thus there are no other symmetries.

Theorem 5.10. The homeomorphism $\varphi$ maps the pair $\left(\mathbb{C P}^{2}, t^{+}\right)$to the pair $\left(\mathbb{C P}^{2}, t^{-}\right)$.

Proof. We apply Algorithm 2.4

(1) Set $\sigma=\left(L_{1}\right)\left(L_{2} L_{3}\right)\left(L_{4} L_{5}\right)\left(L_{6} L_{10}\right)\left(L_{7} L_{9}\right)\left(L_{8}\right)$.

(2) Set $L_{2}, L_{4}, L_{3}$, and $L_{5}$ as $x=0, x=z, y=0$, and $y=z$, respectively.

(3) This produces the following equations:

$$
\begin{aligned}
& L_{1}: z=0, \quad L_{2}: x=0, \quad L_{3}: y=0, \quad L_{4}: x=z, \quad L_{5}: y=z, \quad L_{6}: y=t x+z, \\
& L_{7}: y=s x+\frac{s}{t} z, \quad L_{8}: y=\left(1-\frac{s}{t}\right) x+\frac{s}{t} z, \quad L_{9}: y=s x-t z, \quad L_{10}: y=t x-t z,
\end{aligned}
$$


where $s^{2}-t s+t^{2}=0$, or $s^{ \pm}=\frac{1 \pm \sqrt{3} i}{2} t$.

(4) Setting $t=1$ we get $s^{2}-s+1=0$, or $s^{ \pm}=\frac{1 \pm \sqrt{3} i}{2}$. It is easy to check that $\varphi\left(L_{i}^{+}\right)=L_{\sigma(i)}^{-}$, where $s^{+} \mapsto s^{-}$because $\left(s^{+}\right)\left(s^{-}\right)=1$. We apply Randell's Isotopy Theorem to the rest of each component.

\section{Some cases from the literature which pose problems for Algorithm 2.4}

Although this Algorithm 2.4 can be applied without a problem for the cases above, it does not appear to be applicable as stated for some other cases, specifically the Falk-Sturmfels arrangement and Rybnikov's example, which actually gives a Zariski pair.

We include these counterexamples here, in the hopes that they will lead to understanding what conditions might be necessary in order to ensure that this technique holds in general.

Example: Falk-Sturmfels arrangement. Now consider the Falk-Sturmfels arrangement (cited as unpublished in [CS97]) of nine lines. Like the arrangements above, it has a quadratic defining equation. However its roots are real (and Galois conjugate) and not complex (conjugate).

We start with the combinatorics of the arrangement obtained from the geometry in Example 5.2 of [NY12]. We give a configuration table for the points of higher multiplicity in Table 10 .

\begin{tabular}{|cccc|cccc|c|}
\hline$L_{1}$ & $L_{2}$ & $L_{3}$ & $L_{4}$ & $K_{1}$ & $K_{2}$ & $K_{3}$ & $K_{4}$ & $H_{9}$ \\
\hline$q$ & $q$ & $q$ & $q$ & $e_{1}$ & $e_{2}$ & $e_{3}$ & $e_{4}$ & $e_{1}$ \\
$e_{1}$ & $e_{2}$ & $e_{3}$ & $e_{4}$ & $e_{6}$ & $e_{5}$ & $e_{5}$ & $e_{6}$ & $e_{2}$ \\
$e_{5}$ & $e_{6}$ & $e_{7}$ & $e_{8}$ & $e_{8}$ & $e_{7}$ & $e_{8}$ & $e_{7}$ & $e_{3}$ \\
& & & & & & & & $e_{4}$ \\
\hline
\end{tabular}

TABLE 10. A configuration table for the points of higher multiplicity of the FalkSturmfels arrangement.

Proposition 6.1. The Falk-Sturmfels arrangement has $\mathbb{Z}_{4}$ as its automorphism group with a $\mathbb{Z}_{2}$ subgroup.

Proof. Any symmetry of the arrangement must respect the lines $L_{1}, L_{2}, L_{3}, L_{4}$ that pass through the lone quadruple point labeled $q$ in the configuration table. A permutation on the set $\left\{L_{1}, L_{2}, L_{3}, L_{4}\right\}$ of lines will also respectfully act on the sets $\left\{e_{1}, e_{2}, e_{3}, e_{4}\right\}$ and $\left\{e_{5}, e_{6}, e_{7}, e_{8}\right\}$ of triples also on these lines. Of course this fixes the line $H_{9}$.

By the first set of triples, this permutation must also respectfully act on the set $\left\{K_{1}, K_{2}, K_{3}, K_{4}\right\}$ of lines containing these triples. This must also respectfully act on the set $\left\{\left\{e_{6}, e_{8}\right\},\left\{e_{5}, e_{7}\right\}\right.$, $\left.\left\{e_{5}, e_{8}\right\},\left\{e_{6}, e_{7}\right\}\right\}$ of pairs of remaining triples.

The following shows that no permutation except for the identity may fix an element of the set $\left\{e_{5}, e_{6}, e_{7}, e_{8}\right\}$, each of which is the same up to symmetry. Suppose, for example, that the triple $e_{5}$ is fixed. Then the line $L_{1}$ is fixed, and the lines $K_{2}, K_{3}$ may either be acted on by the identity or a transposition. Supposing it is a transposition, then $\left(K_{2} K_{3}\right)$ gives $\left(e_{2} e_{3}\right)\left(e_{7} e_{8}\right)$, which leads to $\left(L_{3} L_{4}\right)$, a contradiction.

Let us suppose now that the permutation sends the triple $e_{5}$ to $e_{7}$. Then this sends the line $L_{1}$ to $L_{3}$ and the triple $e_{1}$ to $e_{3}$. This forces the unordered pair of lines $\left\{K_{2}, K_{3}\right\}$ to be sent to the set $\left\{K_{2}, K_{4}\right\}$.

Suppose by way of contradiction that the line $K_{2}$ is sent to itself, and so the line $K_{3}$ is sent to the line $K_{4}$. Then the triple $e_{7}$ must be sent back to $e_{5}$, and the triple $e_{3}$ must be sent to $e_{4}$. This would send the line $L_{3}$ to $L_{4}$, forcing the triple $e_{7}$ to be sent to $e_{8}$, instead, a contradiction. 
Thus we may assume this permutation sends the ordered pair of lines $K_{2}, K_{3}$ to the ordered pair of lines $K_{4}, K_{2}$. This sends the triple $e_{2}$ to $e_{4}$, the triple $e_{7}$ to $e_{6}$, the triple $e_{3}$ to $e_{2}$, and the triple $e_{8}$ to $e_{5}$. This gives the permutation $\left(L_{1} L_{3} L_{2} L_{4}\right)\left(K_{1} K_{3} K_{2} K_{4}\right)\left(e_{1} e_{3} e_{2} e_{4}\right)\left(e_{5} e_{7} e_{6} e_{8}\right)$ yielding a $\mathbb{Z}_{4}$ symmetry.

The inverse of this permutation sends the triple $e_{5}$ to $e_{8}$, as expected. The only other remaining option is to send the triple $e_{5}$ to $e_{6}$, and this yields a $\mathbb{Z}_{2}$ symmetry that lives within the $\mathbb{Z}_{4}$ symmetry above.

Remark 6.2. In [NY12, Example 5.2], Nazir and Yoshinaga make use of this $\mathbb{Z}_{4}$ combinatorial symmetry of the Falk-Sturmfels arrangement without recognizing it. They use this symmetry to show that it is not a Zariski pair.

However the $\mathbb{Z}_{2} \subseteq \mathbb{Z}_{4}$ symmetry does not give the two disconnected components of the moduli space! In fact, the $\mathbb{Z}_{2}$-action on the moduli space is trivial. Thus Algorithm 2.4 does not apply as it is currently stated to this case.

Example: Rybnikov's arrangement. This example comes from taking two copies of the MacLane arrangement and identifying three lines from each.

As stated below Example 1.10 of [ABCRCAMB06, the group of symmetries for Rybnikov's arrangement is $S_{3} \times \mathbb{Z}_{2}$.

Remark 6.3. Rybnikov's example is, as we mentioned at the start of this paper, a Zariski pair, and thus no symmetry should exist to allow us to identify components of the moduli space.

However there is a $\mathbb{Z}_{2} \subseteq S_{3} \times \mathbb{Z}_{2}$ symmetry subgroup of the automorphism group. Thus Algorithm 2.4 does not apply as is currently stated to this case. The authors suspect this has something to do with having two copies of the MacLane arrangement.

\section{REFERENCES}

[AB94] Enrique Artal Bartolo, Sur les couples de Zariski, J. Algebraic Geom. 3 (1994), no. 2, $223-247$.

[ABCRCAMB06] Enrique Artal Bartolo, Jorge Carmona Ruber, José Ignacio Cogolludo Agustín, and Miguel Ángel Marco Buzunáriz, Invariants of combinatorial line arrangements and Rybnikov's example, Singularity theory and its applications, Adv. Stud. Pure Math., vol. 43, Math. Soc. Japan, Tokyo, 2006, pp. 1-34. MR 2313406 (2008g:32042)

[ABCRCAMB05] Enrique Artal Bartolo, Jorge Carmona Ruber, José Ignacio Cogolludo Agustín, and Miguel Ángel Marco Buzunáriz, Topology and combinatorics of real line arrangements, Compos. Math. 141 (2005), no. 6, 1578-1588.

[ACSTY13] Meirav Amram, Moshe Cohen, Hao Sun, Mina Teicher, and Fei Ye, A notion of distance between real arrangements and examples, in preparation, 2013.

[ACTY13] Meirav Amram, Moshe Cohen, Mina Teicher, and Fei Ye, Moduli spaces of ten-line arrangements with double and triple points, arXiv:1306.6105, 2013.

[ATY13] Meirav Amram, Mina Teicher, and Fei Ye, Moduli spaces of arrangements of 10 projective lines with quadruple points, Adv. in Appl. Math. 51 (2013), no. 3, 392-418.

[CS97] Daniel C. Cohen and Alexander I. Suciu, The braid monodromy of plane algebraic curves and hyperplane arrangements, Comment. Math. Helv. 72 (1997), no. 2, 285-315.

[Grü09] Branko Grünbaum, Configurations of points and lines, Graduate Studies in Mathematics, vol. 103, American Mathematical Society, Providence, RI, 2009.

[Mac36] Saunders MacLane, Some Interpretations of Abstract Linear Dependence in Terms of Projective Geometry, Amer. J. Math. 58 (1936), no. 1, 236-240. MR 1507146

[NY12] Shaheen Nazir and Masahiko Yoshinaga, On the connectivity of the realization spaces of line arrangements, Ann. Scuola Norm. Sup. Pisa XI (2012), no. 4, 921-937.

[Ran89] Richard Randell, Lattice-isotopic arrangements are topologically isomorphic, Proc. Amer. Math. Soc. 107 (1989), no. 2, 555-559.

[Ryb11] G. L. Rybnikov, On the fundamental group of the complement of a complex hyperplane arrangement, Funktsional. Anal. i Prilozhen. 45 (2011), no. 2, 71-85. 
[Ye13] Fei Ye, Classification of moduli spaces of arrangements of 9 projective lines, Pacific J. of Math. 265 (2013), no. 1, 243-256.

[Zar13] Anna Zarkh, Geometric visualization of braid monodromy and applications, Master's thesis, BarIlan University, 2013.

Department of Mathematics, Bar-Ilan University, Ramat-Gan, 52900, Israel and Shamoon College of Engineering, Bialik/Basel Sts., Beer-Sheva 84100, Israel

E-mail address: meirav@macs.biu.ac.il, meiravt@sce.ac.il

Department of Mathematics, Bar-Ilan University, Ramat Gan 52900, Israel and Department of Mathematics, Technion, Haifa 32000, Israel

E-mail address: cohenm10@macs.biu.ac.il

Department of Mathematics, Bar-Ilan University, Ramat Gan 52900, Israel and Huazhong Normal University, People's Republic of China

E-mail address: hsunmath@gmail.com, hsun@mail.ccnu.edu.cn

Department of Mathematics, Bar-Ilan University, Ramat Gan 52900, Israel

E-mail address: teicher@macs.biu.ac.il

Department of Mathematics, The University of Hong Kong, Hong Kong

E-mail address: fye@maths.hku.hk

Department of Mathematics, Bar-Ilan University, Ramat Gan 52900, Israel

E-mail address: annazarkh@gmail.com 